\title{
Treatment of Combined Injuries of the Axillary and Suprascapular Nerves with Scapulothoracic Dissociation
}

\author{
Kazufumi Sano ${ }^{1}$ Satoru Ozeki ${ }^{1}$ \\ ${ }^{1}$ Department of Orthopaedic Surgery, Dokkyo Medical University \\ Koshigaya Hospital, Saitama, Japan \\ J Brachial Plex Peripher Nerve Inj 2015;10:e62-e65.
}

\begin{abstract}
Address for correspondence Kazufumi Sano, MD, PhD, Department of Orthopaedic Surgery, Dokkyo Medical University Koshigaya Hospital, 2-1-50 Minami-Koshigaya, Koshigaya, Saitama 3438555, Japan (e-mail: prssano@nifty.com).
\end{abstract}

\begin{abstract}
A 20-year-old man suffered the combined axillary and suprascapular nerve palsies associated with scapulothoracic dissociation by motorcycle accident. The dislocated shoulder girdle was reduced and stabilized with osteosynthesis of the fractured clavicle and reattachment of the trapezius avulsed from the scapular spine for removal of continuous traction force to these damaged nerves. Because of no evidence of recovery on manual muscle test and electromyogram, exploration for these nerves was administered 6 weeks after injury. Although neurolysis of both nerves revealed neural continuity, excessive tension still existed on the suprascapular nerve. It was thought that previous operation in which the shoulder girdle had been reduced and stabilized as

\section{Keywords}

- scapulothoracic dissociation

- infraclavicular hematoma

- axillary nerve

- suprascapular nerve

- brachial plexus injury

- suprascapular notch

- spinoglenoid notch much as possible could not achieve complete anatomical reduction of the scapula. As an additional treatment, medial walls of the suprascapular and spinoglenoid notches were shaven to relax the suprascapular nerve. After a year, complete recovery of both the axillary and suprascapular nerve was identified. Although scapulothoracic dissociation is commonly recognized as massive injury of the shoulder girdle with poor prognosis because of existence of accompanied severe neurovascular injuries, there are more than a few cases in which partial damage on the infraclavicular brachial plexus is only accompanied. In case of them, there is the possibility of lesions in continuity of the nerves in which good prognosis might be expected with surgical intervention including early reduction of the shoulder girdle for removal of excessive tension to the damaged nerve.
\end{abstract}

\section{Background}

Scapulothoracic dissociation (STD) was first described by Oreck et al in $1984^{1}$ as potentially life-threatening condition. Among patients who escaped death, severe disability of the injured upper extremity is common secondary to devastated neurovascular injuries. With increasing recognition of STD, however, it has been understood that severity of accompanied neurovascular injuries is varied in wide spectrum from

received

March 13, 2015

accepted after revision

September 21, 2015

published online

November 3, 2015 no damage to complete rupture of them. ${ }^{2,3}$ Zelle et al proposed classification of severity of STD in relation to accompanied musculoskeletal and neurovascular complications. ${ }^{4}$ Although there are more than a few cases in which partial damage of the brachial plexus is only accompanied, no detailed description of treatment for them has been reported. We report our strategic treatment for a case of STD with partial damage on the infraclavicular brachial plexus with successful result.
Copyright $\odot 2015$ Georg Thieme Verlag KG Stuttgart · New York
License terms

(®) (1) $\Theta$ 

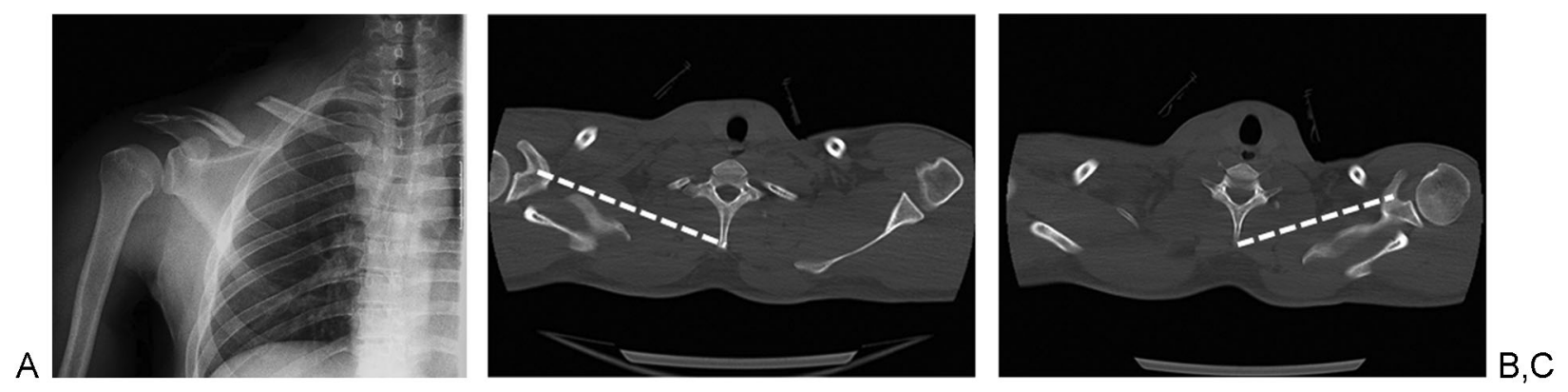

Fig. 1 (A) X-ray showed inferolateral displacement of the scapula with a diaphyseal fracture of the clavicle. (B, C) Scapular index on computed tomography scans. The distance from the apex of the vertebral spinous process to the coracoid base (dotted line on B) divided by that of the uninjured side (dotted line on C) was 1.32 (the average in the normal population: $1.07 \pm 0.04$ ).

\section{Case Presentation}

A 20-year-old man referred with diagnosis of the right STD on a day after motorcycle accident. The patient complained disability of elevation of the right shoulder with severe pain around the shoulder girdle. On physical examination, elevation and external rotation of the shoulder were impossible and local dysesthesia in the skin over a small part of the lateral upper arm (regimental badge area) was recognized. Although manual muscle test (MMT) could not be accessed particularly due to exacerbated pain reflected on the whole arm, moderate active motion of the elbow, wrist, and digits could be identified. An X-ray showed inferolateral displacement of the scapula with a diaphyseal fracture of the clavicle (-Fig. 1A). Scapular index ${ }^{5}$ on computed tomography scans (the distance from the apex of the vertebral spinous process to the coracoid base divided by that of the uninjured side) was 1.32 (the average in the normal population: $1.07 \pm 0.04$ ) (-Fig. 1B, C). Magnetic resource imaging revealed infraclavicular hematoma without detection of any root injuries. With a diagnosis of STD with partial injuries on the infraclavicular brachial plexus nerves (Type 2B in Zelle et al's classification ${ }^{4}$ ), the dislocated shoulder girdle was reduced and stabilized with osteosynthesis of the fractured clavicle anteriorly and reattachment of the trapezius avulsed from the scapular spine posteriorly for removal of continuous tension to the brachial plexus nerves on day 8 after the injury (-Figs. 2 and 3). Although scapular index was improved to 1.05 postoperatively and motor functions of the elbow, wrist, and digits became normal grade of MMT in accordance with subsidence of pain, elevation and external rotation of the shoulder were still impossible. With the diagnosis of combined palsies of the axillary and suprascapular nerves without evidence of spontaneous recovery on both MMT and electromyogram, exploration for these nerves was administered 6 weeks after injury. The patient is placed in the lateral position, and a saber-cut incision was applied from the anterior axillary fold, by way of the middle clavicle to the posterior axilla. After osteotomy of the coracoid process, the identified axillary nerve was covered with dense scar from the bifurcation of the posterior cord to the quadrilateral space. Since meticulous microscopic neurolysis of the axillary nerve revealed lesion in continuity and the deltoid muscle was contracted by an electric stimulus to the axial nerve at the bifurcation from the posterior cord, spontaneous recovery would be expected. The suprascapular nerve was then exposed along its entire course from Erb point to the motor branch of the infraspinatus by detaching the trapezius from the scapular spine, acromion, and clavicle. Although lesion in continuity was also identified on the suprascapular nerve, excessive tension existed on whole course of the nerve without contraction of both the supraspinatus and infraspinatus muscle by an electric stimulus to the nerve (-Fig. 4A, B). It was thought that previous operation in which the shoulder girdle was reduced and stabilized as much as possible could not achieve complete anatomical reduction of the scapula. As an additional treatment, medial walls of the suprascapular and spinoglenoid notches were chiseled to
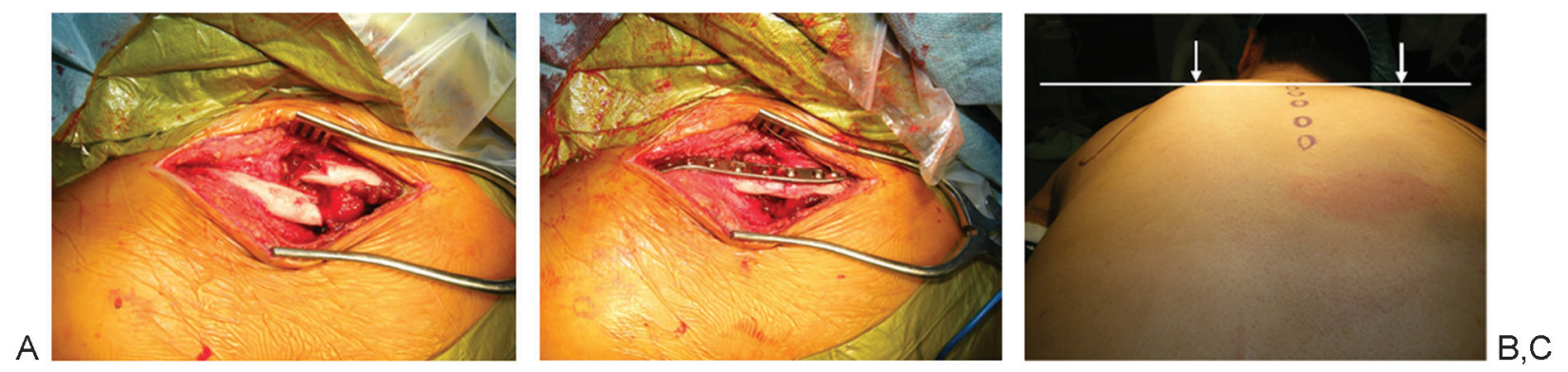

Fig. 2 Repair of anterior component. (A) Exploration of the fractured clavicle. (B) Osteosynthesis of the fractured clavicle. (C) Lateral eccentric position of the scapula (thick arrow) compared with uninjured side (thin arrow) still existed after osteosynthesis of the fractured clavicle. 
A

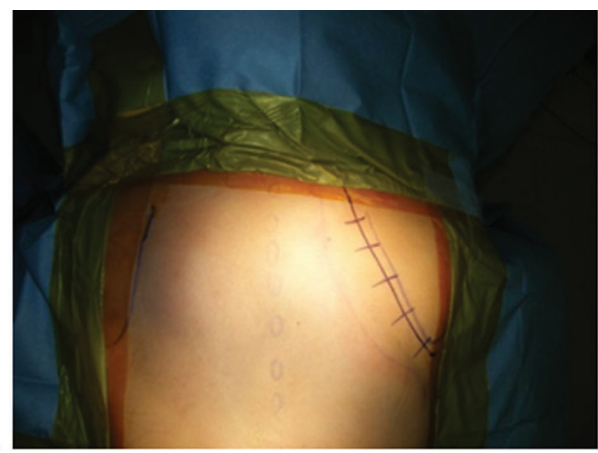

$\mathrm{B}$
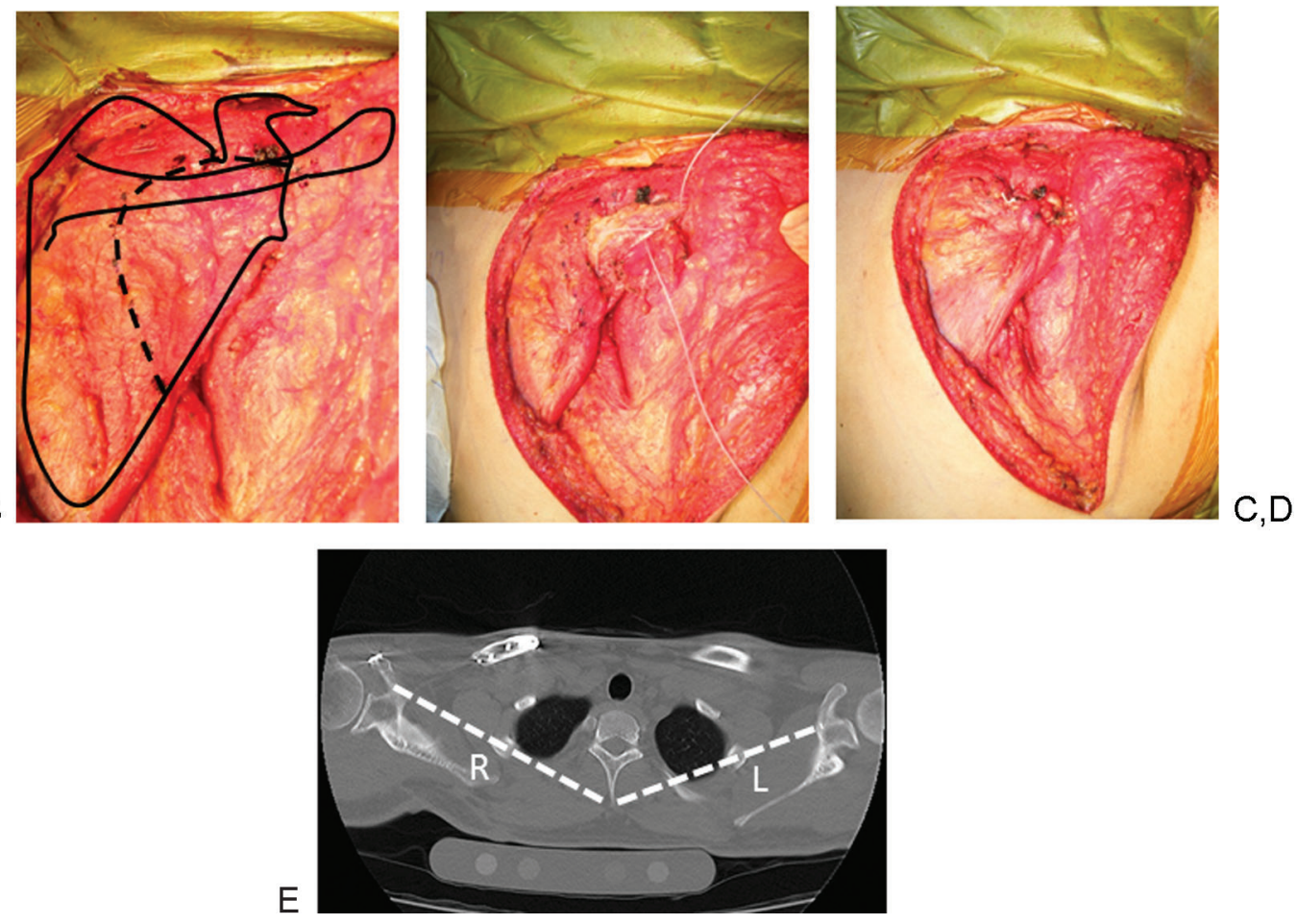

Fig. 3 Repair of posterior component. (A) Incision was made along with the medial edge of the scapula. (B) There was no muscle component but thin and less elastic scar tissue in a shadow area. (C, D) The avulsed trapezius was reattached to the scapular spine using bone anchors. (E) Postoperative scapular index $(\mathrm{R} / \mathrm{L})$ was improved to 1.05 .

release the suprascapular nerve (-Fig. $\mathbf{4 C}$, D). After a year, complete recovery of both the axillary and suprascapular nerve was identified with MMT and electrical evaluation of the deltoid, supraspinatus, and infraspinatus.

\section{Discussion}

STD is the result of either a massive traction force to the upper extremity or massive direct blunt force to the shoulder girdle. $^{2}$

Combined injuries of the axillary and suprascapular nerves are a subtype of the infraclavicular brachial plexus injury, and occur by means of powerful traction force to the shoulder. ${ }^{6}$ Because of same cause of them, combined injuries of the axillary and suprascapular nerves can be accompanied with wide spectrum of STD.

Because of its potentially life-threatening aspect, treatment of STD has been mainly focused on acute phase in which emergency care and advanced resuscitation technique are necessary for the subclavicular vascular injuries and/or concomitant multiple injuries. ${ }^{1,4,7}$ Surgical restorations of the shoulder girdle and related structures are recommended in a couple of weeks, ${ }^{2}$ and anterior restoration consists of open reduction and internal fixation of the accompanied clavicle fracture or the dislocated acromioclavicular or sternoclavicular joint is widely recognized as corresponded treatment. ${ }^{7}$ With only restoration of disrupted anterior components, however, adequate stabilization to the shoulder girdle cannot be expected ( $\mathbf{F i g}$. 2). For ideal stabilization and reduction of the dissociated scapula, avulsed muscles such as the trapezius, rhomboids, and levator scapulae should be reattached to the scapula posteriorly.

Neurovascular injuries accompanied with STD are varied in wide spectrum from no damage to complete rupture of them. Accompanied nerve injuries occur in $94 \%$ of patients and the brachial plexus paralysis is usually complete. ${ }^{8}$ 

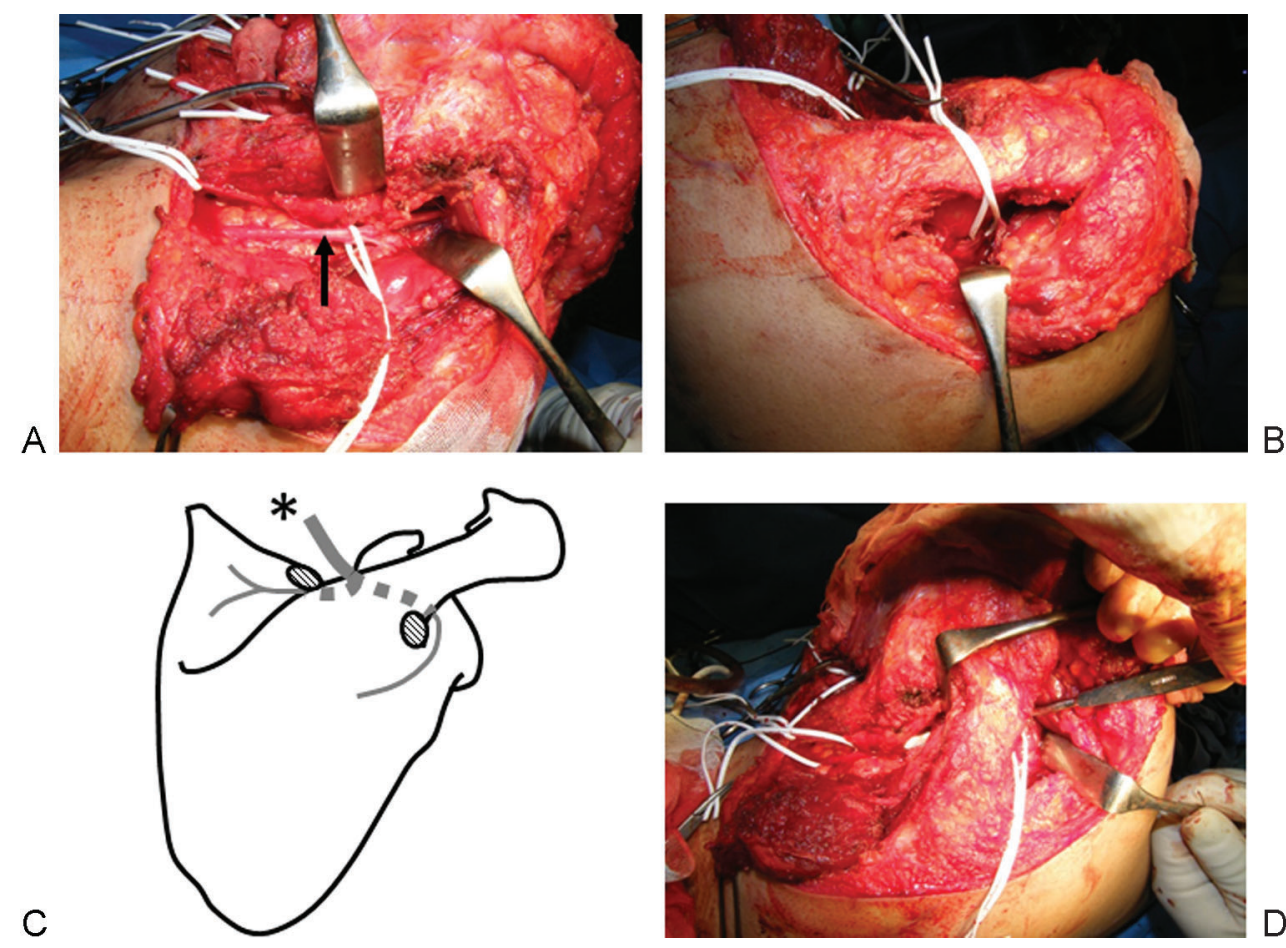

Fig. 4 (A) Excessive tension existed on the suprascapular nerve (arrow). (B) Traction force to the nerve from the suprascapular notch to spinoglenoid notch. (C) The medial walls of the suprascapular and spinoglenoid notches (shadow areas) were chiseled to release the suprascapular nerve (asterisk). (D) After notch plasty, the small elevator could be inserted easily into the notches.

Ebraheim et al reported that partial damage was more frequently observed in infraclavicular lesion, and spontaneous recovery occurred in some cases but this was never complete. ${ }^{3}$ In case of STD, continuous traction force on the brachial plexus exists unless inferolateral displacement of the shoulder girdle is reduced, and undoubtedly has adverse effect on spontaneous recovery of the nerve lesions in continuity.

In this case, although osteosynthesis of the fractured clavicle anteriorly and reattachment of the trapezius posteriorly were reasonable treatment to improve scapular index into normal range, other avulsed muscles such as the rhomboids and levator scapulae might have been repaired as well to achieve complete anatomical reduction of STD. Since reattachment of the whole avulsed muscles is technically difficult, supplemental notch plasty to the suprascapular and spinoglenoid notches is a reasonable solution to remove unfavorable traction force to the nerves of lesions in continuity.

\section{Conclusion}

Since some nerves may sustain in lesions in continuity in case of STD with partial damage of the infraclavicular brachial plexus (Type 2B in Zelle et al's classification), surgical intervention including early reduction of the shoulder girdle for removal of excessive traction force to the damaged nerves should be considered.

\section{Acknowledgment}

We would like to thank Vern Fischer, an English language specialist at Dokkyo Medical University Koshigaya Hospital, for his assistance editing this article.

\section{References}

1 Oreck SL, Burgess A, Levine AM. Traumatic lateral displacement of the scapula: a radiographic sign of neurovascular disruption. J Bone Joint Surg Am 1984;66(5):758-763

2 Althausen PL, Lee MA, Finkemeier CG. Scapulothoracic dissociation: diagnosis and treatment. Clin Orthop Relat Res 2003;(416): 237-244

3 Ebraheim NA, Pearlstein SR, Savolaine ER, Gordon SL, Jackson WT, Corray T. Scapulothoracic dissociation (closed avulsion of the scapula, subclavian artery, and brachial plexus): a newly recognized variant, a new classification, and a review of the literature and treatment options. J Orthop Trauma 1987;1(1):18-23

4 Zelle BA, Pape HC, Gerich TG, Garapati R, Ceylan B, Krettek C. Functional outcome following scapulothoracic dissociation. J Bone Joint Surg Am 2004;86-A(1):2-8

5 Kelbel JM, Jardon OM, Huurman WW. Scapulothoracic dissociation. A case report. Clin Orthop Relat Res 1986;(209):210-214

6 Mikami Y, Nagano A, Ochiai N, Yamamoto S. Results of nerve grafting for injuries of the axillary and suprascapular nerves. J Bone Joint Surg Br 1997;79(4):527-531

7 Lavelle WF, Uhl R. Scapulothoracic dissociation. Orthopedics 2010; 33(6):417-421

8 Damschen DD, Cogbill TH, Siegel MJ. Scapulothoracic dissociation caused by blunt trauma. J Trauma 1997;42(3):537-540 